\title{
De la Nueva Economía Institucional de los Comunes a lo Común como un Modo de Producción
}

Alfonso Giuliani ${ }^{1}$ y Carlo Vercellone ${ }^{2}$

El presente artículo es una traducción ${ }^{3}$ de Giuliani, A., \& Vercellone, C. (2019). From New
Institutional Economics of the Commons to the Common as a Mode of Production. South
Atlantic Quarterly, 118(4), 767-787. https://doi.org/10.1215/00382876-7825600
Cómo citar: Giuliani, A. y Vercellone, C. (2020). De la Nueva Economía Institucional de los
Comunes a lo Común como un Modo de Producción (Traducción de Emilio Cafassi, Agostina
Dolcemáscolo, Martina Lassalle, Ignacio Perrone y Guillermina Yansen). Revista Hipertextos, 8(13),
pp. 21-45. DOI: https://doi.org/10.24215/23143924e009

\section{Resumen}

El objetivo de este artículo es describir el origen, el significado y las cuestiones que están en juego en el debate que se ha desarrollado alrededor de la reciente área de investigación sobre los comunes y reformular la noción de lo común en un contexto post-socialista de superación del capitalismo. Consideraremos, en primer lugar, las contribuciones y limitaciones del enfoque de Ostrom, el cual ha conducido a una cantidad de investigadores a formular planteos alternativos. En segundo lugar, presentamos las teorías de lo común en singular. Luego de un análisis crítico de los aspectos más salientes de la teoría política y normativa de Dardot y Laval (2014), pondremos la atención en la idea neo-operaísta de lo común como un modo de producción. Presentamos el marco teórico y conceptual de esta idea para mostrar cómo lo común constituye un sistema socio-económico que se deriva de las contradicciones internas del capitalismo cognitivo y ofrece una alternativa global a una representación de la sociedad y la economía que se basa en la diarquía Estado-Mercado.

Palabras clave: comunes, común en singular, modo de producción, economía política de los comunes, capitalismo cognitivo.

\footnotetext{
${ }^{1}$ Investigador en el Centre d'Economie de la Sorbonne, Universite, París.

2 Profesor titular de la Universidad de París 8, Francia, Departamento de Cultura y Comunicación. También es miembro del Centre d'Etudes sur les Medias et l'Internalisation (CEMTI) y es investigador asociado del CNRS- Centre d'Economie de la Sorbonne (CES).

${ }^{3}$ La traducción fue realizada por Emilio Cafassi, Agostina Dolcemáscolo, Martina Lassalle, Ignacio Perrone y Guillermina Yansen, y revisada por los autores del texto original.
} 


\begin{abstract}
The purpose of this article is to describe the origin, the meaning, and the issues at stake in the debate that has developed around this recent area of research of the common and reformulate the notion of the common in a post-socialist context of overcoming capitalism. This article will first of all consider the contributions, but also the limits, of Ostrom's approach, which have led a number of researchers to formulate alternative approaches. Secondly, therefore, we will present the theories of the common in the singular. After a critical analysis of the most salient aspects of Dardot and Laval's (2014) political and normative theory, we will turn our attention to the neooperaista idea of the common as a mode of production. We will present the theoretical and conceptual framework of this idea to show how the commons constitutes a social-economic system that is derived from the internal contradictions of cognitive capitalism and offers a global alternative to a representation of society and economics based on the State-Market diarchy.
\end{abstract}

Keywords: commons, common in the singular, mode of production, political economy of the commons, cognitive capitalism

\title{
Resumo
}

O objetivo deste artigo é descrever a origem, o significado e as questões em jogo no debate que se desenvolveu em torno desta recente área de pesquisa do comum e reformular a noção do comum num contexto pós-socialista de superação do capitalismo. Este artigo considerará, em primeiro lugar, as contribuições, mas também os limites, da abordagem de Ostrom, que têm levado uma série de pesquisadores a formular abordagens alternativas. Em segundo lugar, portanto, vamos apresentar as teorias do comum no singular. Depois de uma análise crítica dos aspectos mais salientes da teoria política e normativa de Dardot e Laval (2014), vamos voltar nossa atenção para a idéia neo-operaista do comum como modo de produção. Apresentaremos o quadro teórico e conceitual dessa idéia para mostrar como o comum constitui um sistema sócioeconômico que deriva das contradições internas do capitalismo cognitivo e oferece uma alternativa global para uma representação da sociedade e da economia baseada na diarquia Estado-Mercado.

Palavras-chave: comum, comum no singular, modo de produção, economia política do comum, capitalismo cognitivo 


\section{Introducción}

Comenzando con los trabajos fundantes de Elinor Ostrom y la Escuela de Indiana durante los ' 80 s y los ' $90 \mathrm{~s}$, el redescubrimiento del tema de los comunes ha ido codo a codo con una multitud de estudios que definitivamente han enriquecido el pensamiento tanto en la esfera empírica como teórica. Sin embargo, la vitalidad de este nuevo campo de investigación, que cubre el abanico de las ciencias sociales, ha revelado al mismo tiempo notables divergencias. Estas se vinculan tanto a los fundamentos epistemológicos como ontológicos de lo común y su devenir, esto es, el rol que podría jugar en términos de la transformación social.

Estas divergencias son evidentes en la terminología misma adoptada por diferentes académicos. Siguiendo a Ostrom, la nueva economía institucional emplea el término "comunes" exclusivamente en plural con el objetivo de dar cuenta de la pluralidad de las formas institucionales que regulan el funcionamiento de la economía. En contraste, otros enfoques, que se centran en elementos que subvierten el capitalismo, originados en la contra-revolución neoliberal, insisten en usar el concepto de común en singular entendido como un principio general para reorganizar la economía y la sociedad.

El objetivo de este artículo es describir el origen, el significado y las cuestiones que están en juego en el debate que se ha desarrollado alrededor de esta reciente área de investigación, aunque intentando ir más allá del legado de Ostrom y reformular la noción de lo común en un contexto post-socialista de superación del capitalismo. Con eso en mente, este artículo considerará, en primer lugar, las contribuciones, y también las limitaciones, del enfoque de Ostrom, el cual ha conducido a una cantidad de investigadores a formular planteos alternativos. En segundo lugar, presentaremos las teorías de lo común en singular. Luego de un análisis crítico de los aspectos más salientes de la teoría política y normativa de Dardot y Laval (2014), pondremos nuestra atención sobre la idea neo-operaísta de lo común como un modo de producción. Presentaremos el marco teórico y conceptual de esta idea para mostrar cómo lo común constituye un sistema socio-económico que se deriva de las contradicciones internas del capitalismo cognitivo y ofrece una alternativa global a una representación de la sociedad y la economía que se basa en la diarquía Estado-Mercado. 


\section{Contribuciones y cuestiones irresueltas del Nuevo Enfoque Institucional de los comunes de Elinor Ostrom}

Ostrom ha jugado indudablemente un rol pionero en el resurgimiento del tema de los comunes en la teoría económica y la ciencia política. El trabajo de la ganadora del Premio Novel de Economía, ganado junto a Oliver Williamson, otro exponente de la Nueva Escuela Institucional, realizó una decisiva contribución al cuestionar las afirmaciones sobre la ineluctable "tragedia de los comunes" (Hardin, 1968) y la idea según la cual los comunes se encuentran estructuralmente condenados a una auto-destrucción ambiental y económica a menos que sean regulados por el Estado o privatizados.

Empezando por una crítica interna del paradigma neoclásico, Ostrom introdujo la noción de recursos comunes (CPR, por su sigla en inglés) ${ }^{4}$ permitiendo un considerable enriquecimiento de la tipología samuelsoniana tradicional de los bienes públicos y privados. Esto demostró la posibilidad de superar la dicotomía simplista estado-mercado, dividiéndola en dos contribuciones principales: la aplicación de la teoría del conjunto de derechos [bundle of rights theory] para establecer una clara distinción entre res nullius de acceso abierto, y CPRs gobernados, en cambio, por formas específicas de propiedad y regulación de acceso a los recursos; la identificación de una serie de principios de gestión institucionales que permiten la reproducción y la sostenibilidad de los comunes.

A pesar de estas contribuciones, su enfoque continúa viciado por numerosas limitaciones que no le permiten formular lo común como un verdadero modo de producción alternativo a la lógica del capitalismo. En particular, el resurgimiento de los comunes como un potencial tercer modelo de propiedad y gestión de recursos, junto con los modelos privado y público, pone en duda la hegemonía de la economía capitalista de mercado. Ostrom se limita a "solo reconocer el hecho de que hay otras actividades por fuera del mercado que crean valor y que es, por tanto, necesario tomarlas en consideración" (Loveluck 2015: 222) como una dimensión complementaria y solo parcialmente alternativa. No es casual que las configuraciones de los comunes estudiadas por Ostrom correspondan esencialmente a experiencias en el nivel micro-social y

\footnotetext{
${ }^{4}$ Nota de los traductores: la sigla proviene del término "common-pool resources" (CPR) y puede ser también traducido como "recursos de uso común". En términos de Ostrom, el término refiere a recursos (naturales o artificiales), cuya cantidad es suficientemente grande como para hacer difícil o costosa (aunque no imposible) la exclusión de potenciales beneficiarios que obtengan beneficios de su uso (Ostrom, 1990: 30).
} 
comunitario sin considerar condiciones para extenderlas de forma más amplia a la economía.

Esta dificultad de considerar los comunes como algo más que un tercero, intruso en la tradicional representación de la economía, está basado en tres principales límites epistemológicos y analíticos. El primero es que Ostrom permanece atada a una representación samuelsoniana de la economía que se basa en las características intrínsecas de los bienes, aunque efectivamente introduce clarificaciones conceptuales significantes. En particular, en lugar del tradicional concepto de rivalidad, introduce el concepto de substractabilidad. Esta noción permite establecer una gradación de la rivalidad y hacer más elástica la frontera entre los diferentes bienes, en comparación con la tipología neoclásica estándar. Además, poner el acento en la substractabilidad de los bienes se adecúa más a los problemas ambientales típicos de los bienes comunes de la tierra que estudia Ostrom (1990: 32).

Sin embargo, sobre esta base, para Ostrom los únicos bienes, o más precisamente recursos, considerados como comunes son aquellos que son difíciles de excluir y que son rivales, o que tienen alta substractabilidad, tales como las tierras de pastoreo, bosques y pesquerías.

Por supuesto, el enfoque de Ostrom se define por una tipología puramente naturalista de bienes comunes en la medida en que ella insiste en el hecho de que son también, y sobre todo, una construcción social, que es el producto de una acción colectiva. No obstante, esto no parece desplazar una tipología de los comunes limitada a dos tipos de recursos. En primer lugar, los llamados bienes basados en la tierra rivales y no excluibles, que, como mencionamos, son declarados como los únicos bienes comunes auténticos. Los bienes de información y el conocimiento codificado fueron posteriormente añadidos a estos. Hay que señalar que, sin embargo, estos últimos siguen siendo considerados en principio como bienes públicos puesto que son no rivales y difíciles de excluir (Tabla I). Estos recursos son a menudo incluidos en los CPRs, pero esto es solo por las medidas de privatización y el cercamiento del código, lo cual suscita preguntas sobre su inclusión dentro de la categoría de bienes públicos. 
Tabla $\mathbf{n}^{\mathbf{0}} \mathbf{I}$. Tipos de bienes

\begin{tabular}{lll}
\hline & SUBSTRACTABILIDAD & \\
\hline EXCLUSIÓN & Baja & Alta \\
\hline \multirow{2}{*}{ Difíil } & Bienes públicos & Recursos comunes (CPRs) \\
& Conocimiento útil & Bibliotecas \\
& Atardeceres & Sistemas de regadío \\
\hline \multirow{2}{*}{ Fácil } & Bienes de pago o de club & Bienes privados \\
& Suscripciones de revistas & Ordenadores personales \\
& Guarderías & Rosquillas \\
\hline
\end{tabular}

Fuente: Hess y Ostrom, 2009: 11.

De este modo, en la teoría de Ostrom, el desarrollo de los comunes del conocimiento no aparece como la expresión de un movimiento fundamental hacia la auto-organización de la producción conducida por la expansión de una inteligencia colectiva. En cambio, se presenta ante todo como el resultado de una reacción defensiva por parte de la sociedad cuando se enfrenta a nuevos cercamientos del conocimiento. Este esquema compuesto por dos bienes parece atribuir a los comunes un rol esencialmente subsidiario comparado a las fallas respectivas del mercado y el Estado. La verdadera raison d'étre de los comunes del conocimiento desaparecería en gran parte si la lógica neoliberal se pusiera en cuestión y fuera introducido un estilo social-demócrata de regulación.

La segunda limitación concierne a la persistente adhesión al individualismo metodológico, aun cuando este sea revisitado integrando la hipótesis de los comportamientos cooperativos y altruistas y la comunicación entre sujetos, a diferencia de los modelos clásicos de la teoría de juegos. Pero la atenuación de las hipótesis más rígidas del modelo homo oeconomicus no libera a Ostrom de otras limitaciones del enfoque neoclásico.

El rol de las estructuras sociales y económicas en las que los comunes se desarrollan nunca se aborda: se limita a una representación atomista de la sociedad basada en una multitud de individuos situados, en principio, en pie de igualdad. Sobre esta base, el pasaje de la tragedia a lo que podríamos llamar el milagro de los comunes permanece parcialmente anclado a un juicio sobre la mala o buena naturaleza de los instintos y comportamientos humanos. Desde este punto de vista, es revelador que la crítica ostromiana de los argumentos presentados en "La tragedia de los comunes" de Hardin (1968) nunca se centra en refutar históricamente el análisis, en particular, respecto de la 
responsabilidad que cargan los cercamientos por la sobre-explotación de las tierras de pastoreo.

La tercera limitación concierne a la exclusión de las relaciones de poder, de propiedad y de explotación reales que están enmarañadas alrededor de la organización de las relaciones de producción. Esta limitación es particularmente evidente cuando Ostrom analiza las formas de propiedad común. Según Ostrom, la definición de un común no excluye en modo alguno la existencia de una jerarquía social y de profundas desigualdades entre los comuneros [commoners]. Utilizando la teoría del conjunto de derechos [bundle of rights theory] como base, Ostrom argumenta que hay toda una gradación de formas híbridas posibles para organizar los CPRs, que pueden ir entre dos extremos: uno corresponde a una situación en la que los derechos se distribuyen igualitariamente entre todos los participantes y evita cualquier forma de apropiación y transferencia para el uso privado de los recursos. Desde nuestro punto de vista, este es el modelo más cercano a la idea normativa de democracia de los comunes y de propiedad basada en lo común. El otro es donde el común se basa solo en una distribución limitada de ciertos derechos de uso sin erosionar la primacía de los atributos esenciales de la propiedad (gestión, transferencia) bajo su lógica capitalista. Aunque es claro que estas dos configuraciones son irreconciliables, la posición de Ostrom permanece prisionera, también respecto de este punto, de una profunda indecisión. El resultado es un enfoque que, por ejemplo, es incapaz de decirnos cómo la lógica de lo común es diferente de la empresa capitalista y cómo podría representar una alternativa. Frente a la ausencia de un análisis de las relaciones sociales de producción, la impresión es que Ostrom, con su teoría de la propiedad como un conjunto de derechos, a veces halla un camino para ubicar toda forma de distribución de los derechos de propiedad que no se corresponde con los principios estrictos del individualismo propietario en la categoría de propiedad común.

De este modo, en ciertos pasajes, ella va tan lejos hasta considerar la sociedad anónima como una forma de propiedad común en el sentido de que la distribución de las acciones entre los accionistas la hace propiedad compartida. Desde la perspectiva de Schlager y Ostrom (1992), y de Ostrom y Hess (2010: 72), hay incluso un grado de semejanza entre el régimen de propiedad de los comunes y el de una corporación:

La corporación moderna es frecuentemente pensada como el epítome de la propiedad privada. Mientras que comprar y vender acciones del stock 
corporativo es un claro ejemplo de los derechos de la alienación en el trabajo [work] $]^{5}$, las relaciones dentro de una firma están lejos de ser derechos de propiedad 'individuales'. Puesto que el ingreso que será compartido entre los accionistas, los gerentes, y los empleados es en sí mismo un fondo común a ser compartido.

De esto se desprenden dos corolarios importantes:

a) Ostrom parece no poder ver el modo por el cual la diseminación de los derechos de propiedad no mitiga, sino que acentúa su propósito social: extraer plusvalía del trabajo [labor] de los trabajadores sujetados a la gestión de la empresa que personifica los intereses de los dueños de acciones. Ahora, como indican algunos teóricos próximos a la teoría de Ostrom (Coriat 2015; Weinstein 2013), a menos que lo vaciemos de todo su significado preciso, es claro que el modelo de una corporación es exactamente el opuesto al del común.

b) La imposibilidad de decirnos lo que la corporación podría ser como un "común productivo" y por ende la negación a pensar en la extensión de los comunes más allá de la esfera económica en relación con la gestión de un número limitado de recursos.

Debería estar claro que el principal límite del enfoque de Ostrom es no poder entender que lo común es un modo de producción genuino basado en relaciones sociales muy específicas. Este límite lleva a la autora a sostener, muchas veces contradiciéndose a sí misma, una serie de argumentos confusos. La insistencia en los principios de gobernanza pueden así ir de la mano, como se ha mencionado, de un análisis que en un extremo relega lo común a un conjunto predeterminado de bienes, y, en el otro extremo, extiende el concepto

\footnotetext{
${ }^{5}$ El equipo de traducción de Hipertextos se confronta permanentemente con dificultades relativas a la distinción anglosajona entre work y labour en inglés o werk y arbeiten alemán. En efecto, a pesar de que la palabra labour tiene una raíz latina (laborem), cuando se introduce en la lengua inglesa en el siglo XIV, queda ya asociada inequívocamente al sufrimiento, al padecimiento y la aspereza. Como reconoce Raymond Williams, labour designa formas de organización del trabajo bajo relaciones de clase, o en otros términos, relaciones de poder en las que el trabajador no domina y dirige su fuerza de trabajo o es compelido a trabajar por otros. Aquello que Marx, apelando a Hegel, designa con el término "alienación” y entiende la unidad de estas formas de alienación como explotación de la fuerza de trabajo. Como reconoce uno de los traductores de Williams, la traducción como labor resulta insatisfactoria. Otro tanto sucede con la expresión germana arbeit, que deriva del término arba que significa esclavo. Inversamente tanto work como werk aluden a la creatividad, a la ejecutividad y si se nos permite la hipótesis antropológica, a la realización humana. Ante la recurrencia nada casual de esta díada contradictoria entre los artículos que la nuestra revista pone a disposición de los lectores de lengua española, el equipo de traducción de Hipertextos, seguirá intentando utilizar un significante en español que mejor se adapte al espíritu del texto, pero indicando entre paréntesis el término originalmente utilizado por los autores (Véase la traducción de Allmer, 2018, en Hipertextos, 6(10)).
} 
infinitamente hasta que se convierte en una simple forma de distribución de derechos de propiedad compatible con el modelo capitalista de una sociedad anónima por acciones. En este marco, conceptos como común, comunes, bienes comunes, propiedad común, etc., corren el riesgo de perder toda coherencia tanto en términos teóricos como políticos. La coherencia entre estos conceptos emerge claramente, por contraste, cuando lo común es considerado como un modo de producción basado en la auto-gestión y en principios que conciernen a la inapropiabilidad de los medios de producción y el producto del trabajo [labor], que claramente excluyen relaciones de explotación.

\section{Enfoques sobre lo común en singular y la idea de lo común como un modo de producción}

Según el pensamiento de Ostrom, los comunes solo pueden existir en plural. Son una excepción que no puede ir más allá de la esfera micro económica. Su rol es sacar a la luz el pluralismo de los mecanismos de coordinación y gobernanza, sin que implique "un cuestionamiento de la forma de regulación del capitalismo... y sus consecuencias sociales" (Lomazzi 2018:222). En contraste con esta visión, se desarrollaron enfoques sobre lo común en singular ${ }^{6}$ principalmente en Francia. Para estos enfoques, lo común no es ni un conjunto predeterminado de bienes ni un tercero entrometiéndose entre el Estado y el mercado, sino un principio general de organización de la sociedad. Más allá de este elemento de acuerdo, hay diferencias fundamentales entre la noción de lo común esencialmente "política" y jurídica de Dardot y Laval (2014) y la que se desarrolla en el marco de las teorías neo-operaístas del capitalismo cognitivo. Antes de continuar con nuestra presentación de lo común como un modo de producción, parece apropiado, entonces, resumir los rasgos característicos y los puntos de desacuerdo con el marco teórico de Dardot y Laval.

\subsection{Lo común en singular como un principio político}

Según Dardot y Laval (2014:23) lo común en singular sería esencialmente un principio político de transformación social basado en la co-obligación que surge de la co-actividad y del proceso de "agregación" [pooling]. Con esta base, "la

\footnotetext{
${ }^{6}$ Un momento importante en su presentación fue el seminario Du Publica u Commun organizado en París entre 2010 y 2013 por Pierre Dardot, Christian Laval, Antonio Negri, Judith Revel y Carlo Vercellone.
} 
estrategia de institución política de lo común se caracteriza por cómo involucra el establecimiento de un derecho al uso fuera de la propiedad, la relativización del Estado gracias a la institución autónoma de una ley del común y su objetivo de sustituir a la democracia representativa con una democracia de participación" (Sauvetre 2016:5).

Sin embargo, en una jugada aparentemente sorprendente, Dardot y Laval simultáneamente afirman que lo común en singular no es de ningún modo un nuevo modo de producción (Dardot y Laval 2014:582). Hay de hecho una contradicción teórica entre estas dos ideas, dado que no está claro cómo lo común puede tomar la forma singular, como un principio revolucionario de la auto-institución de la sociedad, sin sentar las bases de un sistema económico y social alternativo, es decir, lo que Marx llamó un modo de producción.

En particular, respecto a lo común siendo definido como una co-actividad, para estos autores no habría un sujeto colectivo o productivo que pre-exista y pueda constituir la base de lo común. Este sujeto solo puede ser el resultado, ex post, de la institución política de lo común. Esto sería particularmente cierto dado que el trabajo cognitivo [labor], para estos autores, no posee autonomía y está dominado por el capital a través de métodos que son aún más abarcativos que aquellos de la era Fordista. Se afirma que la subsunción real ha dado un nuevo salto cualitativo, asumiendo la misma subjetividad del trabajo [labor] y cancelando cualquier posibilidad de implementar un proceso de reapropiación de la producción. Como resume Vibert (2016:6), lo común aparece así "como encadenado a una co-actividad sin anclaje cultural o histórico". Dardot y Laval "tienden de este modo a opacar las relaciones entre lo común como una praxis instituyente y la situación socio-histórica” (Lomazzi y Ménard 2018:82).

Pese a que es definido como una co-actividad, toda la atención se centra en el momento político y jurídico que instituye a lo común, sin identificar a los sujetos de producción social que, en nuestra opinión, tienen la facultad de establecerlo y hacer de esto una virtualidad inscrita en las contradicciones de la relación capital/trabajo propias de una economía basada en el conocimiento.

El problema es que, de este modo, el enfoque de Dardoy y Laval se desliza hacia una suerte de idealismo ${ }^{7}$, en el sentido noble de la palabra, pues ya no queda claro de qué condiciones materiales puede emerger políticamente lo común. De hecho, es la idea de lo común como un principio político que precede tanto su institución como la subjetividad que luego será su simple

\footnotetext{
${ }^{7}$ Este punto fue enfatizado particularmente por Negri (2014).
} 
soporte. Esta noción de lo común es similar a lo que podríamos llamar una utopía sin sujeto, y esta ausencia es el rasgo que, sin saberlo, Dardot y Laval comparten con los enfoques naturalistas sobre los comunes. Mientras que en esos enfoques tenemos, de hecho, un catálogo de bienes comunes definidos independientemente de los sujetos que son capaces de instituirlos y efectivamente producirlos, en Dardot y Laval tenemos una "capacidad de actuar" que es similar a un puro imperativo categórico desconectado de las condiciones históricas mediante las cuales esta fuerza de invención toma forma en la subjetividad del trabajo [labor]. Según la visión utópica y normativa de Dardot y Laval (en términos de lo que debe ser), el enfoque de lo "común como un modo de producción" sustituye a un análisis materialista y positivo de lo común y su formación basado en dos premisas metodológicas.

El primer postulado es que lo común no es un simple principio político, sino que debe ser pensado como una relación social de producción que encuentra su fundamento ontológico históricamente determinado en la autonomía potencial del trabajo cognitivo [labor]. Desde esta perspectiva, es posible distinguir, en su articulación, dos dimensiones de la ontología de lo común. Por un lado, lo que puede llamarse lo común en sí, que corresponde al total de recursos (desde la tierra, la cultura, modos de vida e interacciones sociales y productivas), que pre-existe al capital, aunque el último tiende a asimilarlos a res nullius para transformarlos en lo que Karl Polanyi hubiera llamado mercancías ficticias. Por otro lado, lo que puede llamarse lo común para sí, que está organizado colectivamente y a sabiendas en torno a la fuerza inventiva de la inteligencia colectiva, que tanto en los sectores de alta tecnología como en los llamados tradicionales lleva el antagonismo capital-trabajo al mismo terreno del desarrollo de las fuerzas productivas. Entonces, las relaciones sociales de lo común estimulan las mismas dinámicas de la innovación tecnológica y social, y es precisamente en éstas dinámicas que lo común se muestra inequívocamente como un modo de producción.

La segunda premisa metodológica se aparta del esquema mecanicista que el marxismo convencional ha establecido entre la estructura y la superestructura. Esta es una condición indispensable para captar el vínculo inextricable que cada vez pone más presión en las esferas de la política y la ley por un lado, y las esferas económica y social por el otro lado. El hecho de que la única forma en la cual la crisis de la ley del valor que aqueja al capitalismo cognitivo pueda ser contrarrestada sea mediante un formidable fortalecimiento y extensión de los derechos de propiedad intelectual (DPI) es un ejemplo perfecto. Los DPI son 
lo que permite al capital facilitar la supervivencia de la primacía de la mercancía y capturar plusvalor mediante mecanismos cada vez más rentísticos. Una vez más, como en los albores del capitalismo, es muy difícil distinguir los instrumentos "extra-económicos" de la llamada "acumulación primitiva" de aquellos de la llamada "acumulación ordinaria".

\section{Los principios teóricos y metodológicos de las teorías de lo común como un modo de producción}

La noción de lo común como un modo de producción descansa entonces en el análisis de las transformaciones históricas de la cooperación en el trabajo [work] y, consecuentemente, de la naturaleza de los productos. En este contexto, lo "común", como señalan Hardt y Negri (2009) no es un objeto, una sustancia que precede y trasciende la existencia humana. Lo "común" es la actividad social e históricamente determinada que produce incesantemente nuevas instituciones, que son al mismo tiempo condiciones y el resultado del propio "común”.

Respecto a las teorías económicas de los bienes comunes, hay una doble inversión en términos teóricos y metodológicos.

\subsection{Desde las características intrínsecas de los bienes hasta el trabajo [work] como el fundamento ontológico de lo común}

La primera inversión consiste en cuestionar la idea según la cual la naturaleza intrínseca de ciertos bienes es lo que los hace un bien común, en lugar de uno privado o público.

La relevancia de este concepto es, además, refutada mediante una simple observación de la dinámica real de la economía. Ningún bien está, de hecho, destinado, en función de sus cualidades intrínsecas, a convertirse ipso facto en objeto de un determinado método de gestión. Se pueden brindar distintos ejemplos para respaldar esta afirmación.

Bienes como por ejemplo servicios de salud y educación son teóricamente rivales y excluibles a través de la fijación de precios, en el mismo sentido que los bienes privados. Aun así, son producidos con frecuencia por el Estado o por el tercer sector en la forma de servicios colectivos y no comercializables. Lo opuesto es también igual de cierto. Esto es, ningún valor de uso escapa como tal de la esfera de la producción comercial y de ganancias, como está 
demostrado en la creciente presión ejercida por el sector privado en una variedad de bienes públicos y comunes. El caso del agua es un buen ejemplo. Considerada como un bien común, esta ha sido objeto de un poderoso proceso de privatización, generando numerosos conflictos sociales y políticos en Europa y otros lugares. Por ejemplo, en Italia, en 2011, un referéndum fue aprobado para bloquear el proceso de mercantilización del agua impulsada por varias leyes inspiradas por directivas europeas. A pesar del resultado del referéndum, en muchas ciudades la vuelta a la gestión municipal del agua encuentra todavía fuertes resistencias. Aun en el caso de bienes públicos puros, como la defensa, la justicia y la seguridad, pueden ser producidos por los sectores privados dentro de una lógica de comercialización y ganancias. En los Estados Unidos, una gran parte del servicio carcelario es operado por el sector privado. Otro ejemplo extremo y emblemático en Italia, es la empresa de la Mafia, que en tanto industria privada de protección (Gambetta, 1992), comercializa la función relativa al uso de la "violencia legítima estatal". Otro ejemplo de cómo la clasificación de un bien no determina cómo este es gestionado, es el conocimiento. Por su naturaleza no rival y difícil de excluir, el conocimiento pertenece a la categoría de bienes públicos. En la realidad, su producción y su circulación están lejos de ser aseguradas por el sector público y/o de acuerdo a un modelo no comercial. Por el contrario, el conocimiento hoy en día es sujeto de un poderoso proceso de privatización, llamado por James Boyle (2003) como "el segundo movimiento de cercamiento". En el sentido opuesto, comunidades densas en conocimiento, como son los casos de software libre o Wikipedia, pueden producir conocimiento como bien común, haciéndolo disponible y gratis, de una manera alternativa a la lógica tanto del sector privado como público. De esta manera, no existe un criterio objetivo que trace una línea entre las esferas de lo público, lo privado y lo común basada en la naturaleza de los bienes. Estos límites son el resultado, en cambio, de decisiones políticas que traducen las relaciones de poder y compromisos que, en un momento determinado, se establecen entre los sujetos y los intereses que emanan de cada una de las esferas. Lo común es, por lo tanto, producto de una construcción social e institucional que lo elige a este estatuto. Esto no refiere a una esencia que lo precede, sino a una forma de gobernanza y cooperación vinculada al trabajo [work] que asegura su producción, reproducción, y distribución. Como tal, lo común se corresponde potencialmente con todo tipo de recurso, bien, y servicio, incluso si esto no significa ignorar los problemas particulares de gestión que cada una de estos conlleva. 
Finalmente, el mismo tipo de bienes, jerarquía de necesidades, y maneras de satisfacerlos no son estáticas, sino que, por el contrario, evolucionan en el tiempo. La misma rivalidad o no-rivalidad, si es excluible o no-excluible, el carácter material o inmaterial de numerosos bienes cambia con el desarrollo tecnológico. Así, por ejemplo, la digitalización de libros, música y películas libera la difusión de bienes culturales de su soporte material, haciendo que teóricamente pasen de ser bienes privados (rivales y excluibles) a bienes colectivos (no-rivales, difíciles de excluir, y reproducibles con un tiempo de trabajo y costo marginal tendientes a cero) ${ }^{8}$. Por último, pero no por eso menos importante, esta contextualización histórica es quizás más importante para los sujetos de la producción. En este contexto, se puede considerar cómo en la era fordista, para la teoría económica, la misma hipótesis de una actividad en innovación e investigación que se desarrollara por fuera de las instituciones académicas o de los laboratorios de $\mathrm{I}+\mathrm{D}$ de las corporaciones era inconcebible. Hoy en día, sin embargo, con el desarrollo de una intelectualidad de masas, se afirma cada vez más la proposición según la cual una gran parte de estas actividades descansan sobre redes sociales y comunidades intensivas en conocimiento. Incluso economistas como David y Foray (2002) lo convierten en uno de los principales rasgos que caracteriza la afirmación de la economía basada en el conocimiento. Por esto mismo, no debemos partir de una tipología abstracta de los bienes, sino de las formas concretas e históricas del trabajo [work] como el productor mismo de estos últimos. De hecho, es la capacidad del trabajo [work] de organizarse a sí mismo de una manera alternativa, tanto a la lógica jerárquica de la corporación como a la lógica burocrática del sector público, que determina en última instancia la propensión de una serie de bienes o recursos a ser gestionados de acuerdo a los principios de lo común. Aquí encontramos el fundamento ontológico, histórico, y la precondición en el corazón de lo común que recupera su fuerza en el capitalismo contemporáneo.

\subsection{Lo común como un modo de producción: una nueva perspectiva teórica}

La segunda inversión consiste precisamente en discutir lo común como un modo de producción y no simplemente como bienes comunes o comunes. Para entender el

\footnotetext{
${ }^{8}$ La noción de Jeremy Rifkin (2014) del desarrollo de los comunes descansa casi por completo en este tipo de determinismo tecnológico, que incluiría una cada vez más amplia gama de bienes y servicios, incluida la energía, dentro del campo de bienes no rivales y no excluibles.
} 
significado de esta innovación teórica, debemos recordar primero que un "modo de producción" es presentado como un sistema orgánico que une, de una manera relativamente coherente, un conjunto de elementos que se refuerzan entre sí: 1) una manera de pensar dominante respecto de la organización de la producción y sus objetivos; 2) formas específicas de propiedad de los medios de producción y los productos; 3) mecanismos correspondientes de distribución de la riqueza producida y validación social de la actividad productiva, lo cual también implica una métrica alternativa a la propia ley del valor del capitalismo; 4) una forma específica de desarrollar fuerzas productivas y por lo tanto de la innovación vinculada a esta forma de organizar las relaciones sociales de producción; 5) finalmente, lo común, como todo modo de producción, está incrustado en una "formación social" estructurada por una articulación jerárquica de distintos modos de producción. Dicho esto, definir lo común como un modo de producción significa que este contiene potencialmente las características esenciales de un sistema económico y social genuino del cual, así como con el capitalismo, puede ser extraído una suerte de tipo ideal que muestre su coherencia y sus contradicciones. Sobre estas bases, el concepto de común en singular como un modo de producción puede ser caracterizado en dos niveles principales.

\subsection{El principio general de lo común como modo de producción 9}

En el primer nivel, este concepto indica, así, un principio general de autogobernanza en relación a la producción y la sociedad. Su fundamento reside en la autogestión y la inapropiabilidad de los principales instrumentos de la producción y de los recursos de los que depende la reproducción de una sociedad. Aquí nos encontramos con la primera diferencia esencial con el sistema capitalista, establecido sobre el binomio Estado-Mercado, en el cual la democracia permanece relegada en términos políticos a una democracia representativa y es enteramente separada de la esfera económica, esfera en la que las decisiones estratégicas dependen de la propiedad privada y/o pública, que comparten el principio de la propiedad absoluta.

Lo mismo podría decirse de la diferencia que lo común encarna en relación con los viejos modelos del socialismo real en los cuales la propiedad colectiva de los medios de producción era puramente formal, en tanto "la verdadera

${ }^{9}$ Para conocer más discusiones sobre este concepto, véase Vercellone et al. 2015, Negri, 2016, y Vercellone et al. 2017. 
propiedad económica” (aquello que Bettelheim -1970- llamó “posesión”) era monopolio de una clase burocrática. El resultado fue la reproducción de los mismos modelos productivos de capitalismo industrial basado en la división vertical del trabajo [labor] entre ideas y ejecución. Esta configuración explica, además, la velocidad con la cual la clase burocrática se hizo cargo de la propiedad privada de las principales empresas soviéticas, seguida de una nueva y fantástica fase de acumulación primitiva, que fue la llamada transición de las economías soviéticas a las economías de mercado.

En contraste tanto con el sistema capitalista como con el socialismo real, lo común como un modo de producción hace así que la democracia vuelva a ingresar a la esfera de la economía y de las decisiones estratégicas relacionadas con las siguientes preguntas: ¿cómo se produce? ¿Qué se produce? ¿Para quién? ¿Y para satisfacer qué necesidades?

Más precisamente, considerando a los bienes como un todo y modificando parcialmente la definición que Benkler (2004:1110) utiliza para los bienes comunes informacionales, podemos afirmar que la producción está basada en lo común "cuando nadie utiliza derechos exclusivos para organizar esfuerzos o capturar su valor" y cuando la cooperación en el trabajo [work] se basa en mecanismos sociales que son diferentes comparados con las jerarquías administrativas de la empresa y el objetivo del lucro financiero [financial profitability $]^{1011}$.

\subsection{Las formas de lo común como un modo de producción}

En un segundo nivel, la introducción del concepto de común como modo de producción nos permite aclarar el significado y la articulación de los otros conceptos (comunes, bienes comunes, propiedad común) utilizados en la economía política de los comunes. Al mismo tiempo, los completa teniendo en cuenta otras dimensiones cruciales, como el tipo de desarrollo para las fuerzas

\footnotetext{
${ }^{10}$ A diferencia de la definición de Benkler, introdujimos la noción lucro en lugar de precios como un criterio especial. La decisión se basa en que, si la vocación no mercantil es una de las características de muchos bienes comunes, entonces el criterio de los precios no discrimina. Lo común es, desde nuestra perspectiva, compatible con la producción de bienes destinados al mercado si el objetivo social es no lucrativo. Numerosas organizaciones del llamado tercer sector que son fieles al espíritu del mutualismo y la solidaridad del primer movimiento de trabajadores del siglo XVIII son una prueba de esto, como lo es particularmente el desarrollo reciente del cooperativismo de plataforma (ver Borrits y Nicolini y Paltrineri, en esta publicación). [Nota de los traductores: refiere a la publicación original (véase South Atlantic Quarterly, 118(4))].

11 Nota de los traductores: la expresión "financial profitability" suele ser traducida como rentabilidad financiera. Sin embargo, dado que para los autores el concepto de renta tiene un papel distinguido y muy relevante en el capitalismo actual optamos por traducirlo en este segundo sentido, relativo al lucro.
} 
productivas y las formas de distribución y validación social de la actividad productiva.

En resumen,

1) La noción de comunes en plural designa las expresiones concretas y descentralizadas de este principio general de la organización de la producción, que con métodos específicos y flexibles pueden adaptarse a la gestión de cada tipo de bien, servicio o recurso. Deberíamos recordar, de hecho, que el rechazo de un enfoque que restringe lo común a la naturaleza intrínseca de ciertos bienes, no significa en absoluto ignorar las limitaciones específicas presentadas por la gestión común de diferentes tipos de productos y recursos. Por ejemplo, es evidente que las condiciones de producción de los bienes tangibles estandarizados por un lado, y las condiciones para la producción de l'bumano para y por el humano (salud, educación, trabajos de cuidados, etc.), por otro lado, tienen características profundamente diferentes. En el primer caso, la organización del trabajo [work] puede seguir estando parcialmente ligada a criterios de eficiencia aplicados a la productividad. En cambio, en el contexto de la producción de l'bumano para y por el bumano, la eficiencia es principalmente cualitativa y el concepto mismo de productividad pierde gran parte de su relevancia. Similares consideraciones son válidas en relación con la dimensión de las unidades productivas y la escala de producción. De acuerdo con una línea de pensamiento que encontró uno de los más refinados exponentes en Gorz (1977, 2008), los principios de la autogestión de los comunes sólo pueden llevarse a cabo en pequeñas unidades [productivas] que conducen a la democracia directa y al uso de instrumentos de producción convivenciales. Estos argumentos sin duda conservan un elemento de verdad. Sin embargo, el reconocimiento de la autonomía del trabajo [labor] cognitivo ha inspirado recientemente el desarrollo de nuevas teorías de gestión (Laloux 2015; Robertson 2015) en relación con la autogestión y el autogobierno de las empresas. A pesar de sus contradicciones internas, Thomas Coutrot muestra cómo en estos enfoques ${ }^{12}$ encontramos la solución potencial de un modelo de autogestión para los círculos policéntricos que también es aplicable en las grandes unidades productivas, permitiéndoles reformularlos como un genuino "común productivo" (Coutrot, 2018: 194).

\footnotetext{
12 Debería hacerse notar que estos nuevos enfoques empresariales, en particular el de Robertson, se basan en una teoría de los sistemas fundada en las ideas de Holon y la Holacracia, de la que De Angelis hace gran uso en este número para analizar la dinámica de los comunes. [Nota de los traductores: refiere a la publicación original (véase South Atlantic Quarterly, 118(4))].
} 
2) La noción de bienes comunes designa el conjunto de bienes o recursos producidos y/o administrados por los comuneros, independientemente de sus características específicas en términos de rivalidad y exclusividad, y de materialidad e inmaterialidad. Su rasgo común, lo que nos permite llamarlos bienes, proviene de una doble condición cualitativa. Por un lado, son productos de formas horizontales de cooperación basadas en lo común. Por otro, la riqueza producida no puede ser medida, como lo es con los bienes comerciales, en términos de precios, incluso cuando ciertos comunes, como en el caso de las cooperativas, producen bienes destinados al mercado, pero con un propósito completamente diferente al del lucro.

En este contexto, siguiendo la distinción aplicada por Marx (2009) entre el concepto de valor y el concepto de riqueza ${ }^{13}$ podemos afirmar que la métrica de los bienes comunes es la riqueza social, que cumple al menos tres criterios clave:

- Satisface las necesidades colectivas sobre la base de una utilidad social que no tiene nada que ver con la maximización de la utilidad individual tan querida por el homo economicus y el neoliberalismo.

- Se centra en los bienes y servicios, que a diferencia de muchos otros bienes, desarrolla la capacidad de los individuos, haciendo que, para parafrasear el Manifiesto de Marx y Engels, "el libre desarrollo de cada uno sea la condición para el libre desarrollo de todos"14.

- En el contexto de la relación hombre/naturaleza, respeta una obligación ambiental de reproducir los recursos de generación en generación para cuya medida la huella medioambiental y las emisiones de carbono son indicadores clave.

3) La propiedad común [commons ownership] designa relaciones sociales de propiedad basadas en el uso, la mutualización de recursos y la inapropiabilidad. La cuestión de la propiedad común es sin duda uno de los aspectos más controvertidos y complejos de la definición de lo común como modo de producción. Esto es tanto más cierto cuanto que si la no propiedad define idealmente lo común (Hardt y Negri; Dardot y Laval), este último sigue inmerso en un universo dominado por el capital. En este contexto, una de las

\footnotetext{
${ }^{13}$ Para resumir con dos supuestos: 1) mientras que el valor depende del tiempo de trabajo [labor time] y por lo tanto de la dificultad de la producción, la riqueza depende de la abundancia de valores de uso y, en última instancia, en la no remunerabilidad; 2) la riqueza no sólo proviene del trabajo [work], sino que también es un regalo de la naturaleza que por lo tanto debe ser preservado.

${ }^{14}$ Los indicadores alternativos del PBI (Producto Bruto Interno) derivados de Amartya, la teoría de la capacidad de Sen, como el PNUD (Programa de Desarrollo de las Naciones Unidas) en los índices de desarrollo humano, están actualmente entre los más cercanos a una métrica de la riqueza de lo común.
} 
funciones clave del derecho de lo común, incluyendo el "secuestro" de los instrumentos del derecho privado y público, será en el futuro seguir protegiendo su desarrollo, amenazado por la combinación de las políticas neoliberales y la finalidad extractiva del capitalismo cognitivo.

Hay, en nuestra opinión, tres impulsores principales en el centro de este debate sobre el derecho de lo común ${ }^{15}$.

- El primero, siguiendo el trabajo de la Comisión Rodota (2007) en Italy, es la introducción en el Código Civil de una nueva categoría de bienes que se incluirán en el catálogo de derechos universales inalienables. La noción de bienes comunes de la colectividad se define, por lo tanto, como "cosas que expresan utilidades necesarias para el ejercicio de los derechos fundamentales y el libre desarrollo de la persona" y que, por consiguiente, deben ser salvaguardadas "incluyendo el interés de las generaciones futuras". Esta definición tiene el gran mérito de estar libre de cualquier referencia naturalista ya que no se basa en las características físicas del bien, sino en su propósito social. Esto también permite una clara redefinición de la relación entre lo común y lo Público, si, siguiendo el argumento presentado por Ugo Mattei (2012: VI), consideramos que en lo que respecta a la gestión de estos bienes, lo Público debe ser presentado "como un simple fideicomisario (basado en un mandato o a lo sumo un fideicomiso) y ciertamente no como un propietario, libre de abusarlos, vendiéndolos y privatizándolos indiscriminadamente".

- La segunda idea, en el centro de la experiencia neo-municipal en Nápoles, se basa en la revitalización de la carta de uso cívico de los bienes. Con una decisión aprobada oficialmente el $1^{\circ}$ de junio de 2016 por el consejo municipal a propuesta de los Comuneros, reconoce a una serie de edificios municipales ocupados como "centros de producción” y "espacios, que por su vocación (ubicación territorial, historia y características físicas) se convierten en lugares de uso cívico y colectivo debido a su valor como bienes comunes" 16 . Este es un ejemplo del establecimiento de la propiedad social basado en el uso de un doble interés. En primer lugar, por la relación que establece a través del reconocimiento de la creación por debajo de la ley de lo común. Además, por el paralelismo que establece, en el contexto del derecho y las formas de autogobierno, entre los comunes urbanos en la era del capitalismo cognitivo y los derechos colectivos vinculados a las tierras comunes

\footnotetext{
15 Sobre estas cuestiones véase Brancaccio, Marella, Mattei, y Mancall en este número. [Nota de los traductores: refiere a la publicación original (véase South Atlantic Quarterly, 118(4))].

16 Véase el texto completo de la propuesta en http://www.exasilofilangieri.it/napoli-7-spazi-liberatidiventano-beni-comuni/
} 
antes de su demolición progresiva con la primera fase histórica de la acumulación originaria del capital.

- El tercer impulsor se relaciona con el modelo copyleft y las [licencias] Creative Commons. Estas licencias han utilizado inteligentemente y subvertido los principios de propiedad privada y del copyright desde el interior, para crear un dominio público protegido que se enriquece perpetuamente debido a las propiedades del conocimiento, un dominio en el que "ningún aprovechador [free rider] puede ya intervenir para despojar a los creadores, que es lo que fue permitido por ausencia de derechos, previo al software con una licencia GPL 17" (Coriat 2015: 3). Por supuesto, desafortunadamente las cosas son a menudo más complejas: el copyleft es de hecho mucho menos inviolable y más frágil cuando se enfrenta a las estrategias de las grandes empresas que Coriat parece imaginar. Por esta razón también, la idea de una licencia de copia justa [copy-fair license] que al menos integre una recompensa financiera en caso de que su uso sea comercial está ganando popularidad (Bauwens, 2016).

En cualquier caso, el concepto de dominio público protegido sigue siendo extremadamente fértil para caracterizar la noción de propiedad común, no sólo para los bienes de información, sino también para otros recursos sujetos al principio de escasez. Podríamos definirlo como un conjunto de recursos compartidos al que cada individuo puede acceder y participar en su gestión, tanto contribuyendo a la preservación de los recursos (el caso de los comunes ligados a recursos escasos y/o no renovables) como aumentándolos mediante un uso compartido y creativo (el caso de los comunes inmateriales). En ambos casos, sin embargo, nadie puede sustraer algún elemento para su beneficio privado y en detrimento de la comunidad.

4) En cuanto al tipo de desarrollo de las fuerzas productivas, en lo común existe una estrecha correlación con las relaciones sociales de producción, que al contrario que en el capitalismo, facilitan una noción abierta de conocimiento y tecnología. Esto es lo que muestra con especial claridad el modelo de software libre, el que demostró un nivel de eficacia productiva muy superior al modelo propietario, tanto en términos de calidad del producto como de innovación dinámica. Este círculo virtuoso depende de la articulación coherente entre las

\footnotetext{
${ }^{17}$ Nota de los traductores: por su sigla en inglés "General Public License”.
} 
formas horizontales de organización del trabajo [work] y los modelos de propiedad común, como el copyleft, que permiten la realización de dos condiciones: la participación más amplia de una multitud de colaboradores dispuestos a participar en un proyecto sabiendo que nadie puede apropiarse del producto de su trabajo [work] y la facilitación de la libre circulación del conocimiento y los efectos acumulativos vinculados a su utilización, ya que el software libre, al excluir la propiedad exclusiva, permite a cualquiera acceder a ella de forma gratuita, introducir mejoras y/o lanzar una bifurcación, es decir, un nuevo proyecto para el desarrollo del software libre.

5) Otro aspecto crucial para definir lo común como un modo de producción es el siguiente: lo común, como cualquier otro modo de producción, debe depender de mecanismos de distribución de la riqueza y de validación social de las actividades que apoyen y garanticen su sustentabilidad. Aquí nos enfrentamos a un punto especialmente importante, ya que estos mecanismos son, al menos en este momento, extremadamente frágiles e incompletos. No hay ninguna forma particular de reconocimiento y remuneración del trabajo [work] productivo realizado en el seno de los comunes. Debido a la naturaleza principalmente no mercantil y libre de su actividad (en el sentido de trabajo [labor] que se ofrece tanto libremente como $\sin$ pago), la validación social de la actividad de los comunes sigue dependiendo en gran medida del apoyo público o de la financiación de las partes interesadas asociadas a los grandes oligopolios de la economía digital, cuyo riesgo consiste en comprometer la autonomía del proyecto. El establecimiento de una Ingreso Básico Universal, o mejor aún, lo que llamamos una Ingreso Social Básico (Monnier y Vercellone, 2014), concebido como una renta primaria (vinculada a la producción) y una institución de lo común, sería un primer paso importante para resolver esta carencia. Su justificación debería, por lo tanto, basarse también y principalmente en el reconocimiento de que el trabajo [work] social de los comunes es la fuente de una dinámica de innovación social y productiva que irradia a toda la sociedad. En resumen, significa afirmar que el trabajo [labor] puede ser improductivo de mercancías y beneficios y en cambio producir riqueza no mercantil, por lo que requiere la implementación de un tipo de recompensa en forma de ingresos. 


\section{En conclusión: el contexto de lo común}

La dinámica de lo común es hoy parte de formaciones sociales en las cuales lo Público y lo Privado conviven en una compleja relación de conflicto, competencia e hibridación, pero también a menudo de subordinación. La ausencia de un principio autónomo de financiación y validación social y la dependencia de recursos del sector público y privado son pruebas de estas fragilidades indiscutibles. Así, la cuestión a la que nos enfrentamos hoy consiste en averiguar si, y en qué medida, la difusión de los principios de lo común puede dar lugar a una nueva articulación jerárquica entre lo Común, lo Público y lo Privado, en la que lo común jugará un rol dominante. Hay un elemento esencial necesario para responder a esta pregunta. Actualmente, las dinámicas de los comunes no solo se refieren a las condiciones económicas y sociales que forman parte de lo que Rosa Luxemburgo llamó un exterior pre-capitalista que el capitalismo inevitablemente habría destruido y absorbido. El regreso a la fuerza de los comunes, incluso en formas aparentemente más tradicionales, radica en elementos que las luchas construyeron en los momentos más avanzados del desarrollo capitalista, estableciendo los fundamentos institucionales y estructurales de una economía que se mueve más allá de la lógica del capital. Son lo que podemos definir, al menos potencialmente, como elementos de un exterior post-capitalista. Lo común, así, se presenta a sí mismo, en el sentido marxista, como un nuevo modo de producción en proceso de surgimiento, que se despliega dentro del capitalismo mismo y que puede devenir dominante comparado con las lógicas del Estado y de la economía de mercado capitalista. Reubicándose dentro de la larga historia de las relaciones capital/trabajo [labor], uno de los aspectos clave de la dinámica de auto-organización democrática de los comunes es precisamente generar conflicto, usando nuevas formas y fuerza, al interior del campo de desarrollo de las fuerzas productivas y de una redefinición anti productivista y ecológica de la relación entre hombre y naturaleza.

En suma, ni un simple enclave, ni una mera reacción defensiva de la sociedad en contra de los excesos de los nuevos cercamientos, lo común es la expresión de un movimiento ofensivo real, que construye las condiciones para otra organización económica y social. Dos observaciones finales en este punto son fundamentales para formular el devenir de lo común en el contexto de la superación del capitalismo. La primera es que la oportunidad de transformación de abajo encarnada por el desarrollo de lo común como modo de producción 
resuelve el problema de la transición que caracteriza la tradición socialista, en la que tomar el poder político precede y es la pre condición para un programa de cambio socio económico. La expansión de los principios de democracia directa y auto-organización de lo común reúne estos dos elementos adentro suyo, revirtiendo el sustrato material de las teorías de los dos tiempos (poder político $\rightarrow$ cambio socio económico), ya sean revolucionarias o reformistas.

La segunda es que el potencial de lo común de convertirse en el modelo dominante no significaría, en todo caso, la desaparición de todo vestigio de lo público y lo privado, o del Estado y el mercado. Implicaría, en cambio, el establecimiento de una nueva jerarquía entre lo común, lo privado y lo público. En particular, la potencial supremacía de la lógica de los común no significaría la desaparición de las instituciones del Estado de bienestar y de sus garantías, sino la transformación de su modelo de gestión a través de mecanismos de democracia directa y co-producción, inspirados por lo común, que permitirían la transición desde un modelo estatista hacia un modelo post-burocrático.

\section{Referencias}

Bettelheim, Ch. (1970). Problèmes Théoriques et Pratiques de la Planification (Theoretical and Practical Problems of Planning). Paris: Maspero.

Bauwens, M. (2016). Sauver le monde (Save the World -Toward a Post Capitalist Society with P2P). Paris: Les liens qui libèrent.

Benkler, Y. (2004). Common Based Strategies and the Problem of the Patents. Science, 305 (5687): 1110-11

Boyle, J. (2003) The Second Enclosure Movement and the Construction of the Public Domain. Law and Contemporary Problems, 66: 34-74.

Coriat, B. (2015) (ed.) Le Retour des Communs. La crise de l'idéologie propriétaire (The Return of Commons. The crisis of the proprietary ideology). Paris: Les Liens qui Libèrent.

Coutrot, Th. (2018). Libérer le travail (To Free Work). Paris: Seuil.

Dardot, P. and Laval, Ch. (2014). Common. On Revolution in the 21st Century. Translated by Matthew MacLellan. London: Bloomsbury.

David, P. A . and Foray, D. (2002) Une introduction à I'économie et à la société du savoir (An Introduction to the Economy and Knowledge-Based Society). Revue internationale des sciences sociales, 171: 13- 28.

Comissione Rodotà (2007). Commissione Rodotà -per la modifica delle norme del codice civile in materia di beni pubblici (14 giugno 2007)-Proposta di articolato (Propasal for an amendment of the norms of thc civil code on 
matters of public goods). https://www.giustizia.it/giustizia/it/mg 112 1.wp?contentId=SPS47617 Gambetta, D. (1992). La Mafia Siciliana. Un 'Industria della Proterione Privata (Sicilian Mafia: a Private Security lndustry). Torino: Einaudi.

Gorz, A. (1977). The Division of Labour: The Labour Process and Class Struggle in Modern Capitalism. Hassocks: Harvester Press.

Gorz, A. (2008). Ecology as politics. Translated by Patsy Vidgerman and Jonathan Cloud. Montréal: Black Rose Books Ltd.

Hardin, G. (1968). The Tragedy of the Commons. Science, 62 (3859): I243-48.

Hardt, M. and Negri, A. (2009). Commonwealth. Cambridge, MA: Harvard University Press.

Hess, Ch. and Ostrom, E. (2007) (eds.). Understanding Knowledge as a Commons: From Theory to Practice. Cambridge, MA: MIT Press.

Laloux, F. (2015). Reinventing Organizations: Vers des communautés de travail inspires (Reinventing Organizations: Towards Inspired Work Communities). Diateino: Paris.

Lomazzi, L. (2018). Approches critiques de la captation et de l'exploitation des donnés numérique. Vers une politique du commun? (Critical Approaches to Capturing and Exploiting Digital Data: Toward a Politics of the Common?). In Big Data and Society: Industrialization of Symbolic Mediations. Edited by André Mandoux and Mare Ménard, 211-32. Québec: Presses de I'université du Québec.

Lomazzi, L. and Ménard, M. (2018). Où en est la théorie du/des commun(s)? Vers une économie politique culturelle (Where is the theory of the common(s)? Toward a Cultural Political Economy) tic\&ssociété 12(1): 69-93. 10-40oo/tic\&societe. 2381

Loveluck, B. (2015). Internet, une société contre l'Etat? Libéralisme informationnel et économies politiques de l'auto-organisation en régime numérique (Internet, a Society against the State? Informational Liberalism and Political Economies of Digital Self-organization). Réseaux, 4 (192): 23570. $10.3917 /$ res.192.0235

Marx, K. [1875] (2009). Critique of the Gotha Programme. Gloucester, UK: Dado Press.

Mattei, U. (2012) Beni comuni. Un Manifesto (Common Goods: A Manifesto). Laterza: Roma·Bari. 
Monnier, J-M. and Vercellone, C. (2014). The Foundations and Funding of Basic Social Income as Primary Income. A Methodological Approach. Basic Income Studies, 9 (2): 59-77.

Negri, A. (2016). Il commune come modo di produzione (The Common as a Mode of Production). Euronomade, March 27. www.euronomade.info/?p=7331

Negri, A. (2014). La metafisica del comune (The Metaphysics of the Common). Il Manifesto, May 6. https://ilmanifesto.it/la-metafisica-del-comune/

Ostrom, E. (1990). Governing the Commons. The Evolution of Institution for Collective Action. Cambridge: Cambridge University Press.

Ostrom, E. and Hess, CH. (2010) Private and Common Property Rights. In Encyclopedia of Law and Eeonomics. Edited by Boudewijn Bouckaert, 53-106. Northampton, MA: Edward Elgar.

Rifkin, J. (2014). The Zero Marginal Cost Society: Internet of Things, the Collaborative Commons, and the Eclipse of Capitalism. New York: Palgrave Macmillan.

Robertson, B. J. (2015). Holacracy: The New Management System for a Rapidly Changing World. New York: Henry Holt and Company.

Sauvêtre, P. (2016). Quelle politique du commun? (Which politics of the common?). Sociologies. October 19: 13. https://journals.openedition.org/sociologies/5674

Schlager, E. and Ostrom, E. (1992). Property-Rights Regimes and Natural Resources: A Conceptual Analysis. Land Economics, 68 (3): 249- 62.

Vercellone, C. et al. (2015) Managing the Commons in the Knowledge Economy: Decentralized Citizens Engagement Technologies. Report D3.2, D-CENT. https://dcentproject.eu/wp-content/uploads/2015/07/D3.2-completeENG-v2.pdf

Vercellone, C., Brancaccio, F., Giuliani, A. and Vattimo, P. (2017). Il Comune come Modo di Produzione. Per una critica dell'economia politica dei beni comuni. (The Common as a Mode of Production. Towards a Critique of the Political Economy of Common Goods). Verona: Ombre Corte.

Vibert, S. (2016). L'institution de la communauté (The Institution of the $\begin{array}{lll}\text { Community). SociologieS. October } & \end{array}$ https://journals.openedition.org/sociologies/5683\#quotation.

Weinstein, O. (2013). Comment se construisent les communs: questions à partir d'Ostrom (How the commons are built: Cuestions from Ostrom). In Le retour des communs: la crise de l'idéologie propriétaire, edited by Benjamin Coriat. 69-86. Paris: Editions les Liens qui libèrent. 